\title{
Ultraviolet Beam Focusing in Gallium Arsenide by Direct Excitation of Surface Plasmon Polaritons
}

\author{
Senfeng Lai, Wen Wu, and Wenhua Gu \\ School of Electronic and Optical Engineering, Nanjing University of Science and Technology, Nanjing 210094, China
}

Correspondence should be addressed to Wenhua Gu; guwenhua@njust.edu.cn

Received 4 September 2014; Accepted 11 January 2015

Academic Editor: Nay Ming Huang

Copyright (C) 2015 Senfeng Lai et al. This is an open access article distributed under the Creative Commons Attribution License, which permits unrestricted use, distribution, and reproduction in any medium, provided the original work is properly cited.

This paper proposed that ultraviolet beam could be focused by gallium arsenide (GaAs) through direct excitation of surface plasmon polaritons. Both theoretical analysis and computer simulation showed that GaAs could be a reasonably good plasmonic material in the air in the deep ultraviolet waveband. With a properly designed bull's eye structure etched in GaAs, the ultraviolet electric field could be enhanced to as high as 20 times the incident value, and the full-width-half-maximum (FWHM) of the light beam could be shrunk from $\sim 48^{\circ}$ to $\sim 6^{\circ}$. As a plasmonic material, GaAs was compared to Ag and Al. Within the studied ultraviolet waveband, the field enhancement in GaAs was much stronger than Ag but not as high as Al.

\section{Introduction}

Surface plasmon polaritons (SPPs) refer to the coupling between the incident electromagnetic (EM) wave and the collective free electron plasma wave excited and propagating in the surface part of a specific material [1-10], which is usually called the plasmonic material. Mainly because of the characteristic of intensive EM field enhancement in a small area, especially in the subwavelength scale, the SPP effect has been a hot research topic for years and is widely applied in light beam forming $[2,3]$, subwavelength structure [4], plasma equipment fabrication [5-7], biochemical sensing [810], and other areas. So far, most SPP researches are limited to metal plasmonic materials $[5,11]$. However, some researchers are also actively working on nonmetal plasmonic materials, especially semiconductors [12].

This paper proposed that ultraviolet (UV) SPPs could be directly excited in gallium arsenide (GaAs), one of the most popular semiconductor materials. Using an optimized bull's eye periodic structure, the electromagnetic (EM) field could be enhanced and collimated in $125-261 \mathrm{~nm}$ range.

\section{Theoretical Derivation}

As we know, the SPP wave is the TM wave; using the Maxwell equations and the boundary conditions at the interface where $z=0$, the following equations can be easily derived:

$$
\begin{aligned}
& \frac{k_{z d}}{\varepsilon_{d}}+\frac{k_{z m}}{\varepsilon_{m}}=0 \\
& k_{x m}=k_{x d}=k_{x},
\end{aligned}
$$

where $\varepsilon_{m}$ refers to the relative permittivity of the plasmonic material, $\operatorname{Re}\left(\varepsilon_{m}\right)$ is its real part, $k$ is the wave factor, footnote $x$ stands for $x$ direction which is the surface wave propagation direction, footnote $z$ stands for $z$ direction which is perpendicular to the interface, footnote $m$ stands for the plasmonic material, and footnote $d$ stands for the surrounding dielectric material. And $\varepsilon_{d}$ refers to the relative permittivity of the surrounding dielectric medium.

Use the condition $k_{x}^{2}+k_{y}^{2}+k_{z}^{2}=k^{2}=\varepsilon \omega^{2} / c^{2}$, and consider $k_{y}=0$ in our case; to excite the waves propagating in $x$ direction and attenuating in $z$ direction, $k_{x}$ has to be real, but $k_{z d}$ and $k_{z m}$ have to be imaginary; 


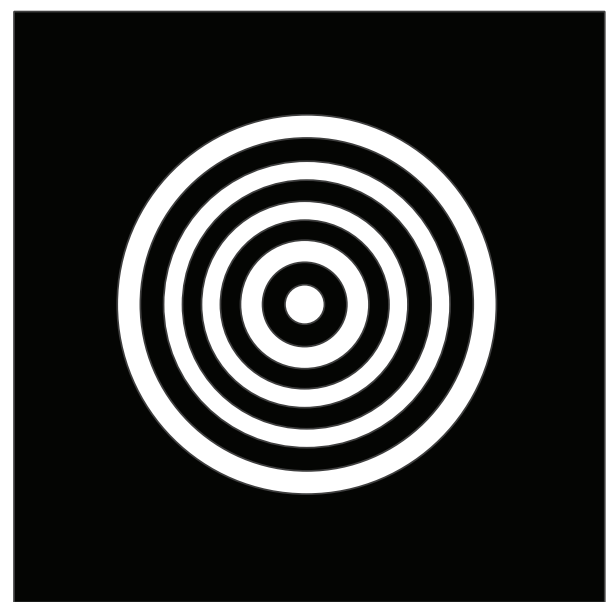

(a)

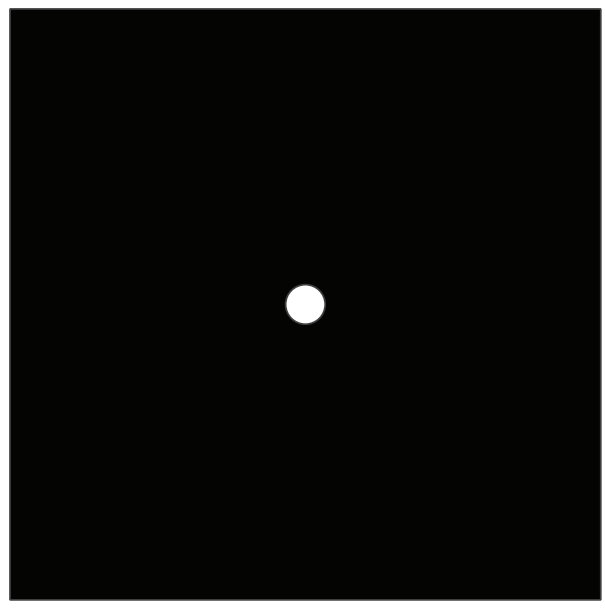

(c)

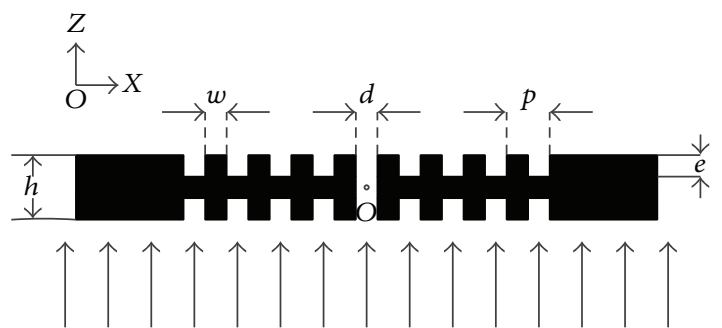

(b)

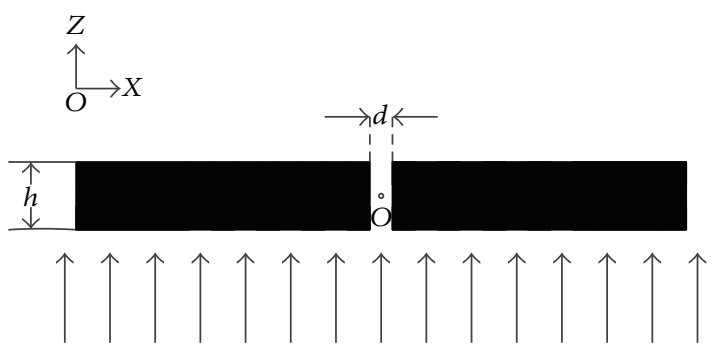

(d)

Figure 1: (a) Schematic diagram of the simulated bull's eye structure, top view. (b) Side view of the bull's eye structure with the incident light and the collection angle illustrated. (c) Schematic diagram of the reference aperture-only structure, top view. (d) Side view of the reference aperture-only structure with the incident light and the collection angle illustrated.

therefore the relative permittivity of the plasmonic material must satisfy the following conditions:

$$
\begin{gathered}
\operatorname{Re}\left(\varepsilon_{m}\right)<0 \\
\left|\varepsilon_{m}\right|>\varepsilon_{d} .
\end{gathered}
$$

Equation (2) is the necessary condition to excite SPP in the plasmonic material [1, 4, 13-20].

Using air as the surrounding medium (relative permittivity equals 1), the relative permittivity of GaAs was calculated and compared with (2). The relative permittivity parameters of GaAs were calculated based on the original data collected from [21]. It was found that (2) could be satisfied in the range of $125-261 \mathrm{~nm}$. So we focused on this deep ultraviolet waveband for possible SPP excitation at the interface of air and GaAs.

\section{Simulation and Results}

Computer simulations using FDTD solutions were designed to study the SPP effect in GaAs. A classic bull's eye structure was used in our simulation. The top view and side view of the simulated bull's eye structure are shown in Figures 1(a) and $1(\mathrm{~b})$, respectively. The plasmonic material formed a thin film with a circular aperture at the center, and a series of periodic concentric-circle shaped grooves were etched from both sides of the thin film. The groove pitch was $p=240 \mathrm{~nm}$, the aperture diameter was $d=120 \mathrm{~nm}$, the groove depth was $e=24 \mathrm{~nm}$ on each side, and the thin film thickness before etching was $h=120 \mathrm{~nm}$. The original point $O$ was set at the center of the film. The surrounding medium was air. It has been well accepted that, in such a structure, surface plasmonic waves can be excited in both surfaces, and the coupling of them could lead to decent EM field enhancement and collimation, provided suitable plasmonic materials and structure parameters are used [2]. As comparison, a reference 


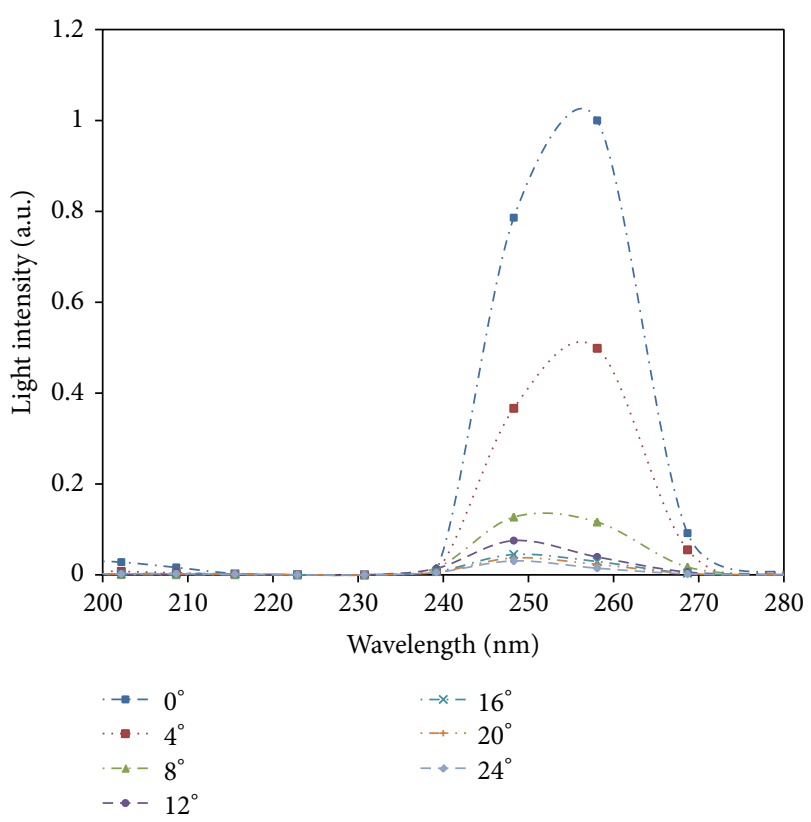

(a)

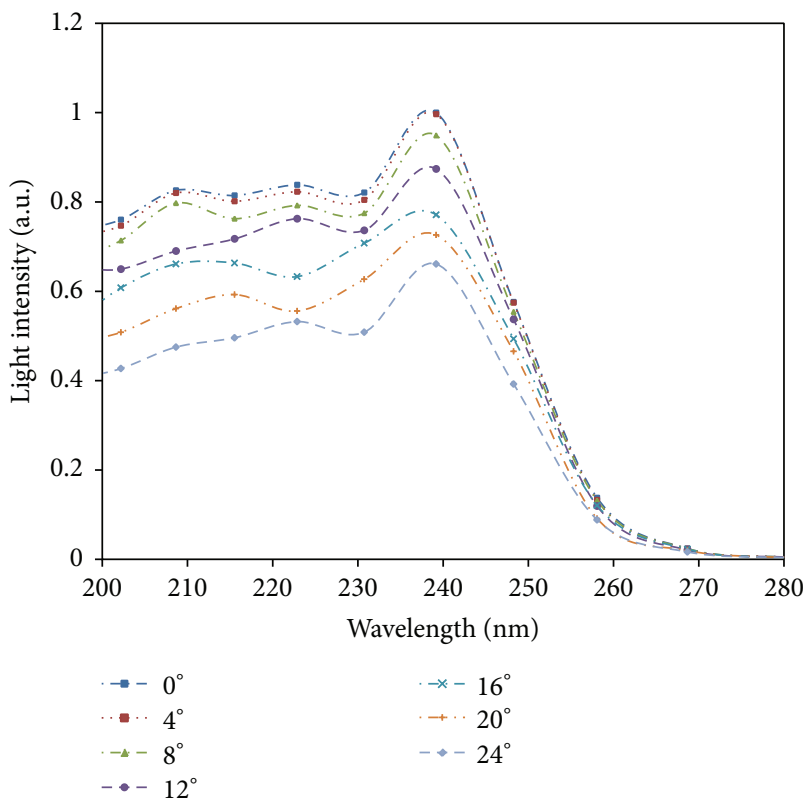

(b)

FIGURE 2: Normalized transmission spectra in the far field at different collection angles for (a) the bulls' eye GaAs structure and (b) the reference aperture-only GaAs structure.

aperture-only structure with the same size but without the periodic grooves was also simulated, as shown in Figures $1(\mathrm{c})$ and $1(\mathrm{~d})$.

The normalized transmission spectra at different collection angles in the far field for the bull's eye structure and the reference structure are shown in Figures 2(a) and 2(b), respectively, and the corresponding angle dependence

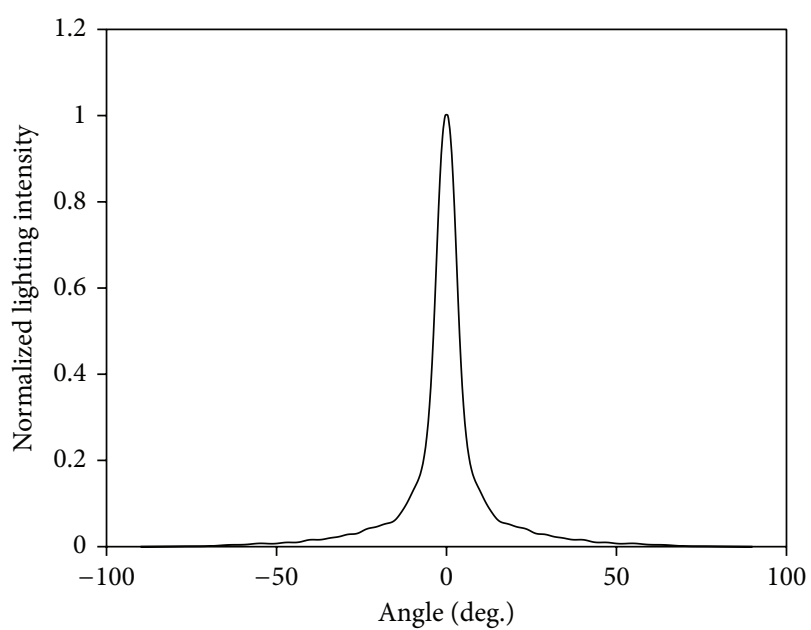

(a)

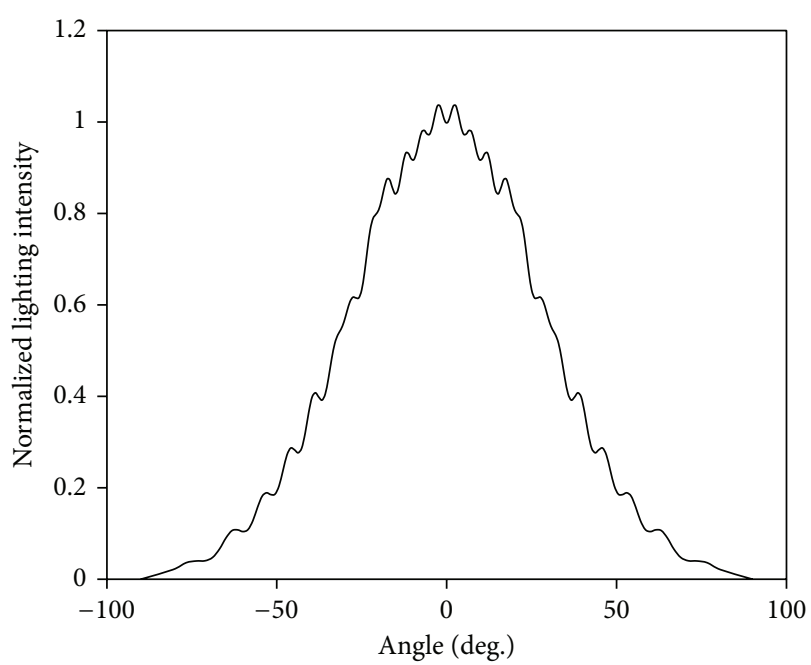

(b)

FIGURE 3: The angle dependence of the transmission light intensity at $248 \mathrm{~nm}$ of (a) the bull's eye structure and (b) the reference apertureonly structure.

at $248 \mathrm{~nm}$ is shown in Figures 3(a) and 3(b). In the reference aperture-only structure, the full-width-half-maximum (FWHM) of the divergence angle was $\sim 48^{\circ}$, and, with the bull's eye structure, the FWHM was shrunk to $\sim 6^{\circ}$, thanks to the SPP effect.

The far field projection maps of the bull's eye structure and the reference structure at $248 \mathrm{~nm}$ incident wavelength are shown in Figures 4(a) and 4(b), respectively. It can be seen that, with the bull's eye structure, the transmitted light diverges much less, and the intensity at the center is about two orders of magnitude higher. For near-field enhancement, the light intensity at the center of the exit surface is 19 times the incident light with the bull's eye structure.

Both the reduced beam divergence and the EM field enhancement at the center are direct proofs of SPP excitation in GaAs. 


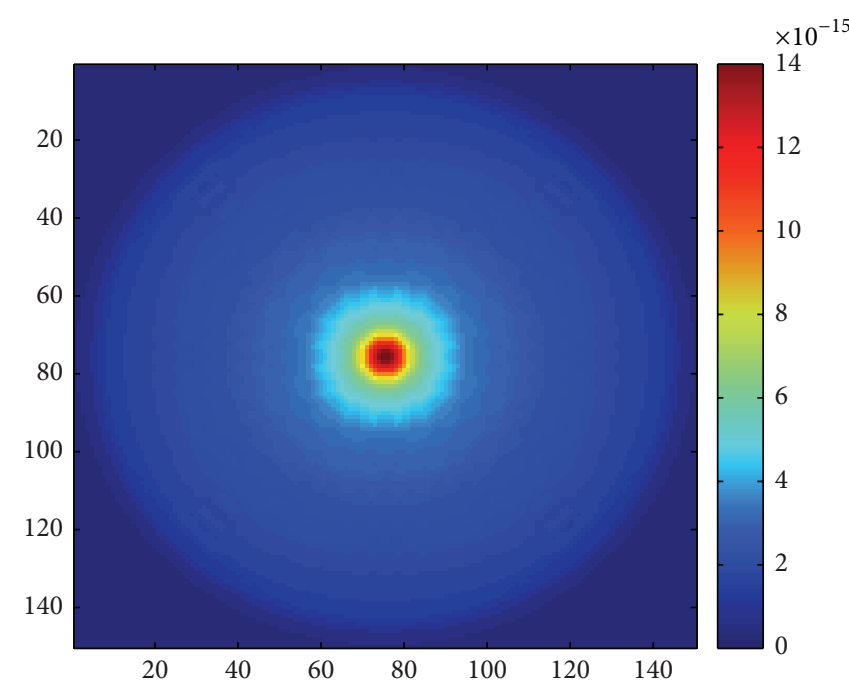

(a)

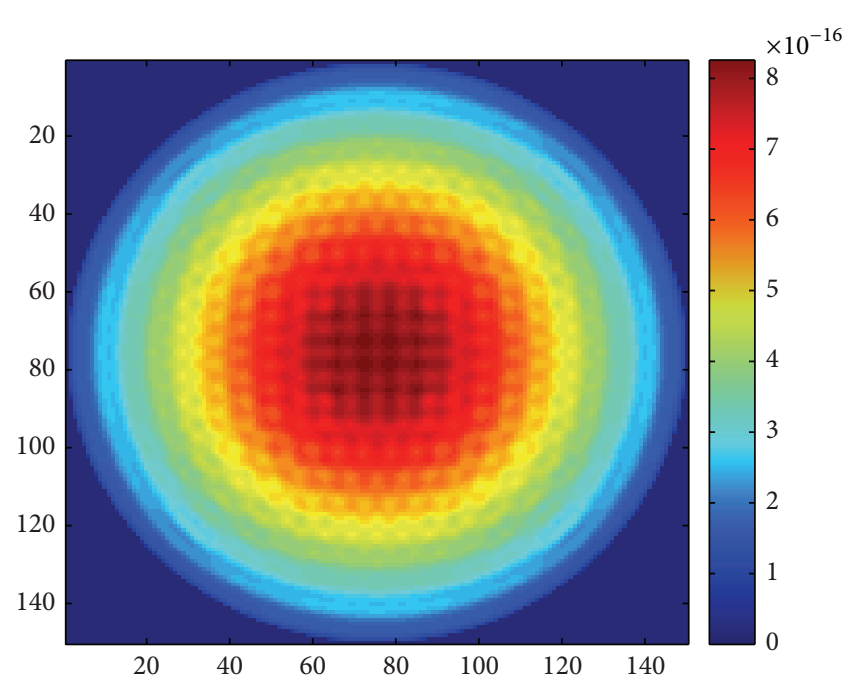

(b)

FIgURE 4: The far field projection map generated at $248 \mathrm{~nm}$ by (a) the bull's eye structure and (b) the reference aperture-only structure.

\section{Analysis and Comparison}

As commonly known good plasmonic materials, Ag and $\mathrm{Al}$ were used for comparison with GaAs. The real and imaginary parts of the relative permittivity of Ag and Al were calculated based on the original refractive index data collected from $[22,23]$. The calculation results were plotted in Figures 5(a) and 5(b) for comparison with GaAs. It is shown that in the waveband of interest the real part of the relative permittivity of GaAs was negative, which satisfies (2) well, while that of $\mathrm{Ag}$ and $\mathrm{Al}$ was generally positive or close to zero. This implies that in the ultraviolet waveband GaAs can be as good as or a better plasmonic material than $\mathrm{Ag}$ and $\mathrm{Al}$.

To further examine the SPP effect of GaAs, another thing to consider is how to quantitatively evaluate the strength of it so as to compare with other materials. The quality factor of SPP, or $Q$, is a reasonable figure-of-merit to evaluate it. Physically, the quality factor $Q$ stands for how well a given plasmonic material can confine the electric field and thus energy within it. For plasmonic materials, $Q$ can be defined as in the following equation [24-26]:

$$
Q=\frac{\left[\operatorname{Re}\left(\varepsilon_{m}\right)\right]^{2}}{\operatorname{Im}\left(\varepsilon_{m}\right)} .
$$

The quality factor curves of $\mathrm{GaAs}, \mathrm{Al}$, and $\mathrm{Ag}$ were thus calculated as shown in Figure 6. It can be seen from Figure 6 that, generally speaking, the quality factor of GaAs is better than $\mathrm{Ag}$ but not as good as $\mathrm{Al}$ in the studied waveband.

The above $Q$ curve comparison provided theoretical reference of the SPP strength for GaAs, Al, and Ag. To verify it by computer simulation, it is preferred to calculate the EM field enhancement quantitatively for all the incident wavelengths in the whole band of $125-261 \mathrm{~nm}$, where SPP excitation in $\mathrm{GaAs}$ is expected. The light intensity at $0^{\circ}$ collection angle at the center of the exit air-film interface is a good parameter to represent the EM field enhancement effect or the SPP effect strength. However, at each incident wavelength, there exists an optimal groove pitch to achieve the highest possible electric field. So we simulated different groove pitch at one wavelength to pick up the pitch yielding the highest light intensity and then plot the curve of light intensity versus wavelength, as shown as the "Intensityactual" curve in Figure 7, to describe the strength of SPP. The result showed that the optimal near-field light intensity could be as high as 20 times the incident light because of the SPP enhancement. The $Q$ curve of GaAs was also plotted in Figure 7 for easy comparison. It can be seen that the shape and peak position of the curves match well with each other.

It is worth pointing out that an easy way to obtain the optimal groove pitch at each wavelength is to relate it with the surface plasmon resonant wave factor, which can be calculated from the following equation [4]:

$$
k_{S P}=k_{0}\left(\frac{\varepsilon_{d} \varepsilon_{m}}{\varepsilon_{d}+\varepsilon_{m}}\right)^{1 / 2},
$$

where $k_{S P}$ is the surface plasmon resonant wave factor and $k_{0}$ is the incident wave factor. Equation (4) was used to calculate the optimal surface plasmon resonant wave factor and thus the corresponding optimal groove pitch $p_{S P}=$ $2 \pi / k_{S P}$ at each incident wavelength between $125 \mathrm{~nm}$ and $261 \mathrm{~nm}$. The simulated structure and mesh grid were scaled in proportion, and then the electric field intensity at $0^{\circ}$ collection angle at the center of the exit air-film interface at each incident wavelength was calculated through simulation. Thus calculated intensity versus wavelength curve is also shown in Figure 7 as the "Intensity-calc" curve. Comparison of the "Intensity-actual" and "Intensity-calc" curves shows that, with the help of (4), one can conveniently estimate the peak wavelength of the SPP strength, yet the intensity could be underestimated. 


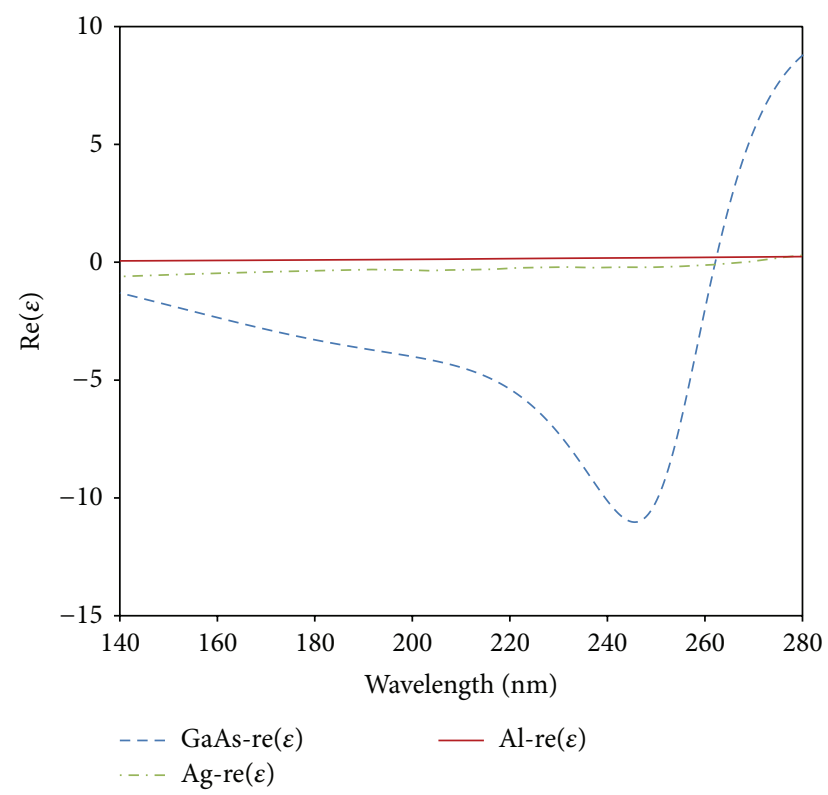

(a)

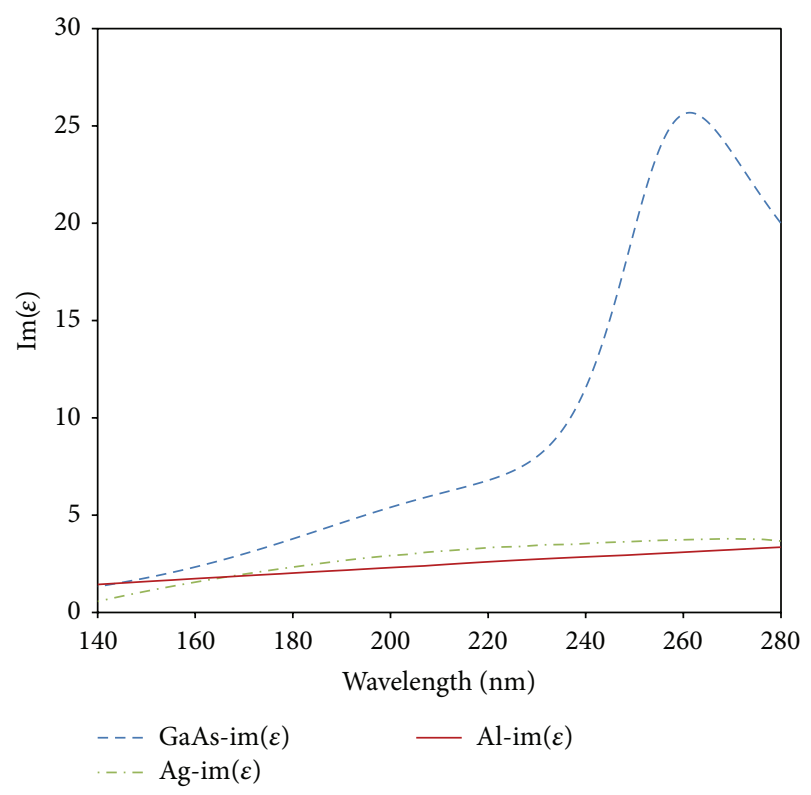

(b)

FIgURE 5: Relative permittivity comparison of GaAs, Al, and Ag.

A similar simulation was run for $\mathrm{Al}$ and $\mathrm{Ag}$ using the same bull's eye structure and groove pitch optimization method. The light intensity enhancement curves at the same unit incident intensity for $\mathrm{GaAs}, \mathrm{Al}$, and $\mathrm{Ag}$ are shown in Figure 8 for comparison. It is shown clearly that GaAs has decent EM field enhancement effect, though not as good as Al. Ag does not show good SPP effect in the studied band. This conclusion complies well with the quality factor comparison result shown in Figure 6.

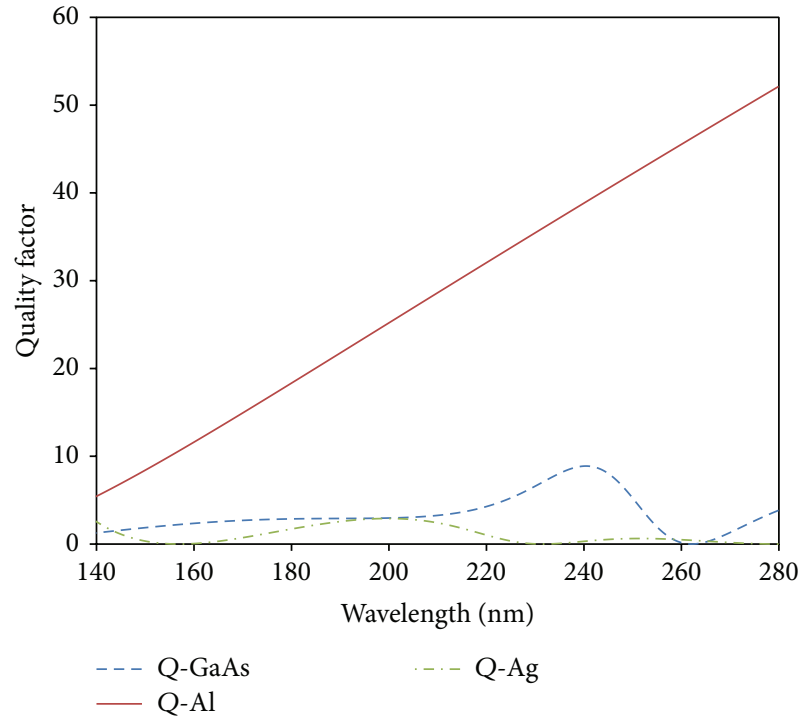

FIguRE 6: Quality factor comparison of GaAs, Al, and Ag.

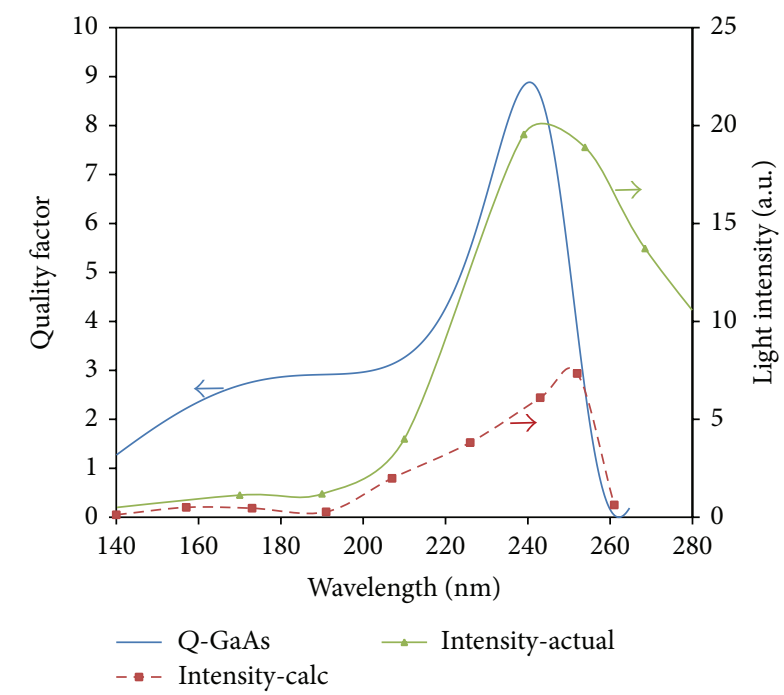

FIGURE 7: Optimal light intensity enhancement spectrum for GaAs, for comparison with the $Q$ spectrum of GaAs. The left vertical axis is the quality factor. The right vertical axis is the light intensity at $0^{\circ}$ collection angle at the center of the exit air-film interface with the optimized groove pitch at each incident wavelength. The green "Intensity-actual" curve is the light intensity simulated with the optimal groove pitch picked up through traversal, while the red "Intensity-calc" curve is the light intensity simulated with the optimal groove pitch calculated by (4).

\section{Conclusion}

To conclude this paper, direct SPP excitation in GaAs material in ultraviolet waveband was proposed, simulated, and optimized. It was shown that, in 125-261 nm waveband, SPPs could be directly excited in GaAs, and decent beam focusing effect could be observed with the optimized bull's 


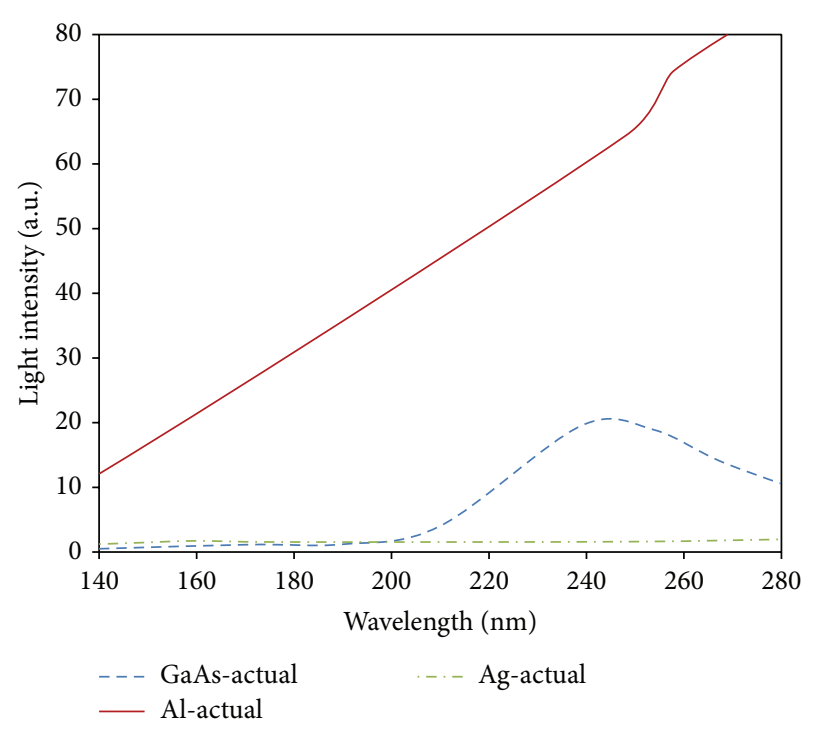

Figure 8: Optimal light intensity enhancement spectra for GaAs, $\mathrm{Al}$, and $\mathrm{Ag}$. The vertical axis is the light intensity field intensity at $0^{\circ}$ collection angle at the center of the exit air-film interface with the optimized groove pitch at each incident wavelength.

eye structure. The computer simulation results match well with the theoretical analysis. How to experimentally prove and make use of these results is an interesting topic of further work.

\section{Conflict of Interests}

The authors declare that there is no conflict of interests regarding the publication of this paper.

\section{Acknowledgments}

This work is partially supported by "the Fundamental Research Funds for the Central Universities," no. 30920130122004. Wenhua Gu thanks the support from the "Star of Zijin" Program of Nanjing University of Science and Technology.

\section{References}

[1] A. V. Zayats, I. I. Smolyaninov, and A. A. Maradudin, "Nanooptics of surface plasmon polaritons," Physics Reports, vol. 408, no. 3-4, pp. 131-314, 2005.

[2] H. J. Lezec, A. Degiron, E. Devaux et al., "Beaming light from a subwavelength aperture," Science, vol. 297, no. 5582, pp. 820$822,2002$.

[3] V. Kravtsov, J. M. Atkin, and M. B. Raschke, "Group delay and dispersion in adiabatic plasmonic nanofocusing," Optics Letters, vol. 38, no. 8, pp. 1322-1324, 2013.

[4] W. L. Barnes, A. Dereux, and T. W. Ebbesen, "Surface plasmon subwavelength optics," Nature, vol. 424, no. 6950, pp. 824-830, 2003.

[5] S. Kawata, Near-Field Optics and Surface Plasmon Polaritons, Springer, Berlin, Germany, 2001.
[6] J. M. Pitarke, V. M. Silkin, E. V. Chulkov, and P. M. Echenique, "Theory of surface plasmons and surface-plasmon polaritons," Reports on Progress in Physics, vol. 70, no. 1, p. 1, 2007.

[7] H. Raether, Surface Plasmons on Smooth Surfaces, Springer, Berlin, Germany, 1988.

[8] N. Liu, T. Weiss, M. Mesch et al., "Planar metamaterial analogue of electromagnetically induced transparency for plasmonic sensing," Nano Letters, vol. 10, no. 4, pp. 1103-1107, 2010.

[9] Z.-G. Dong, H. Liu, J.-X. Cao et al., "Enhanced sensing performance by the plasmonic analog of electromagnetically induced transparency in active metamaterials," Applied Physics Letters, vol. 97, no. 11, Article ID 114101, 2010.

[10] J. Wang, C. Fan, J. He, P. Ding, E. Liang, and Q. Xue, "Double Fano resonances due to interplay of electric and magnetic plasmon modes in planar plasmonic structure with high sensing sensitivity," Optics Express, vol. 21, no. 2, pp. 2236-2244, 2013.

[11] S. Zhang, D. A. Genov, Y. Wang, M. Liu, and X. Zhang, "Plasmon-induced transparency in metamaterials," Physical Review Letters, vol. 101, no. 4, Article ID 047401, 2008.

[12] J. J. Cha, K. J. Koski, K. C. Y. Huang et al., "Two-dimensional chalcogenide nanoplates as tunable metamaterials via chemical intercalation," Nano Letters, vol. 13, no. 12, pp. 5913-5918, 2013.

[13] G. Liang, H. Liang, Y. Zhang et al., "Single-mode surfaceemitting concentric-circular-grating terahertz quantum cascade lasers," Applied Physics Letters, vol. 102, no. 3, Article ID 031119, 2013.

[14] I. V. Konoplev, A. R. Phipps, A. D. R. Phelps, C. W. Robertson, K. Ronald, and A. W. Cross, "Surface field excitation by an obliquely incident wave," Applied Physics Letters, vol. 102, no. 14, Article ID 141106, 2013.

[15] J. S. Gómez-Díaz and J. Perruisseau-Carrier, "Graphene-based plasmonic switches at near infrared frequencies," Optics Express, vol. 21, no. 13, pp. 15490-15504, 2013.

[16] P.-Y. Chen, C. Argyropoulos, and A. Alu, “Terahertz antenna phase shifters using integrally-gated graphene transmissionlines," IEEE Transactions on Antennas and Propagation, vol. 61, no. 4, pp. 1528-1537, 2013.

[17] W. L. Barnes, W. A. Murray, J. Dintinger, E. Devaux, and T. W. Ebbesen, "Surface plasmon polaritons and their role in the enhanced transmission of light through periodic arrays of subwavelength holes in a metal film," Physical Review Letters, vol. 92, no. 10, Article ID 107401, 2004.

[18] M. L. Brongersma and P. G. Kik, Surface Plasmon Nanophotonics, Springer, Berlin, Germany, 2007.

[19] A. V. Zayats and I. I. Smolyaninov, "Near-field photonics: surface plasmon polaritons and localized surface plasmons," Journal of Optics A: Pure and Applied Optics, vol. 5, no. 4, pp. S16-S50, 2003.

[20] K. Okamoto, S. Vyawahare, and A. Scherer, "Surface-plasmon enhanced bright emission from CdSe quantum-dot nanocrystals," Journal of the Optical Society of America B: Optical Physics, vol. 23, no. 8, pp. 1674-1678, 2006.

[21] P. B. Johnson and R.-W. Christy, "Optical constants of the noble metals," Physical Review B, vol. 6, no. 12, pp. 4370-4379, 1972.

[22] E. D. Palik, Handbook of Optical Constants of Solids: Index, Elsevier, 1998.

[23] A. D. Rakić, A. B. Djurišić, J. M. Elazar, and M. L. Majewski, "Optical properties of metallic films for vertical-cavity optoelectronic devices," Applied Optics, vol. 37, no. 22, pp. 5271-5283, 1998. 
[24] M. I. Stockman, "Nanoplasmonics: past, present, and glimpse into future," Optics Express, vol. 19, no. 22, pp. 22029-22106, 2011.

[25] D. J. Bergman and M. I. Stockman, "Surface plasmon amplification by stimulated emission of radiation: quantum generation of coherent surface plasmons in nanosystems," Physical Review Letters, vol. 90, no. 2, Article ID 027402, 2003.

[26] P. R. West, S. Ishii, G. V. Naik, N. K. Emani, V. M. Shalaev, and A. Boltasseva, "Searching for better plasmonic materials," Laser \& Photonics Reviews, vol. 4, no. 6, pp. 795-808, 2010. 

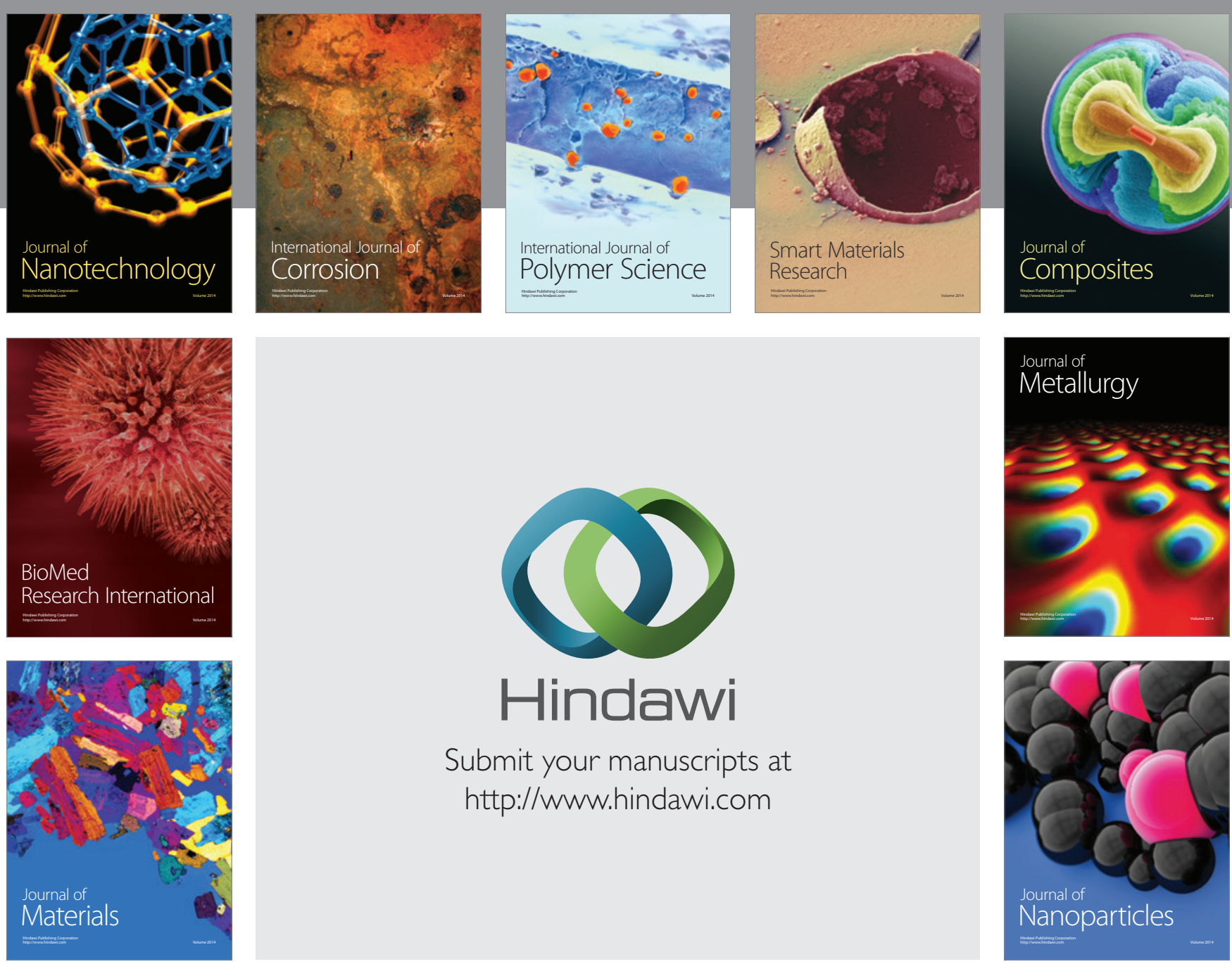

Submit your manuscripts at http://www.hindawi.com
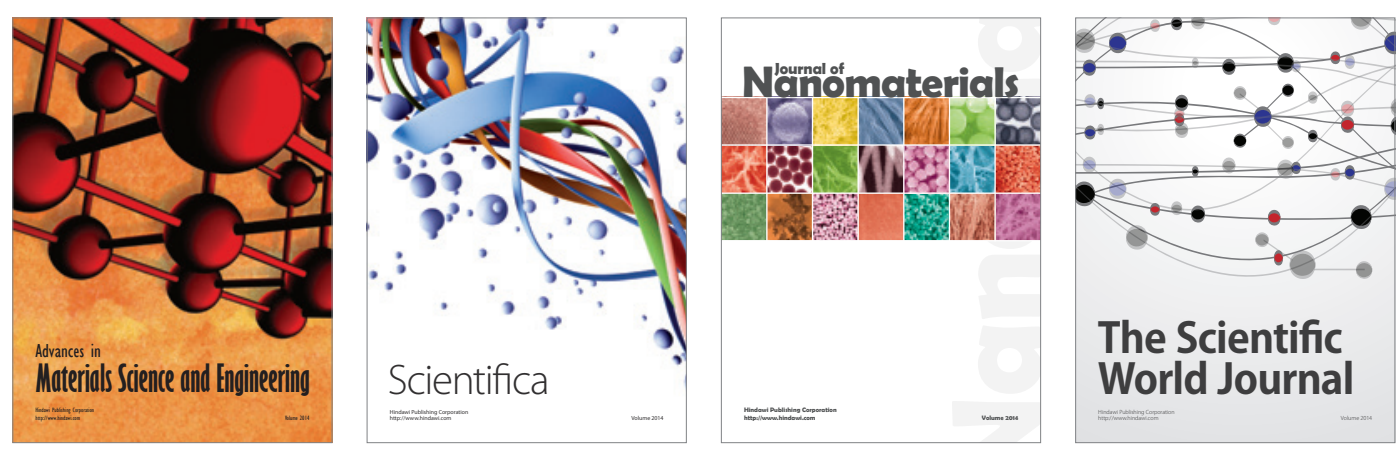

\section{The Scientific World Journal}
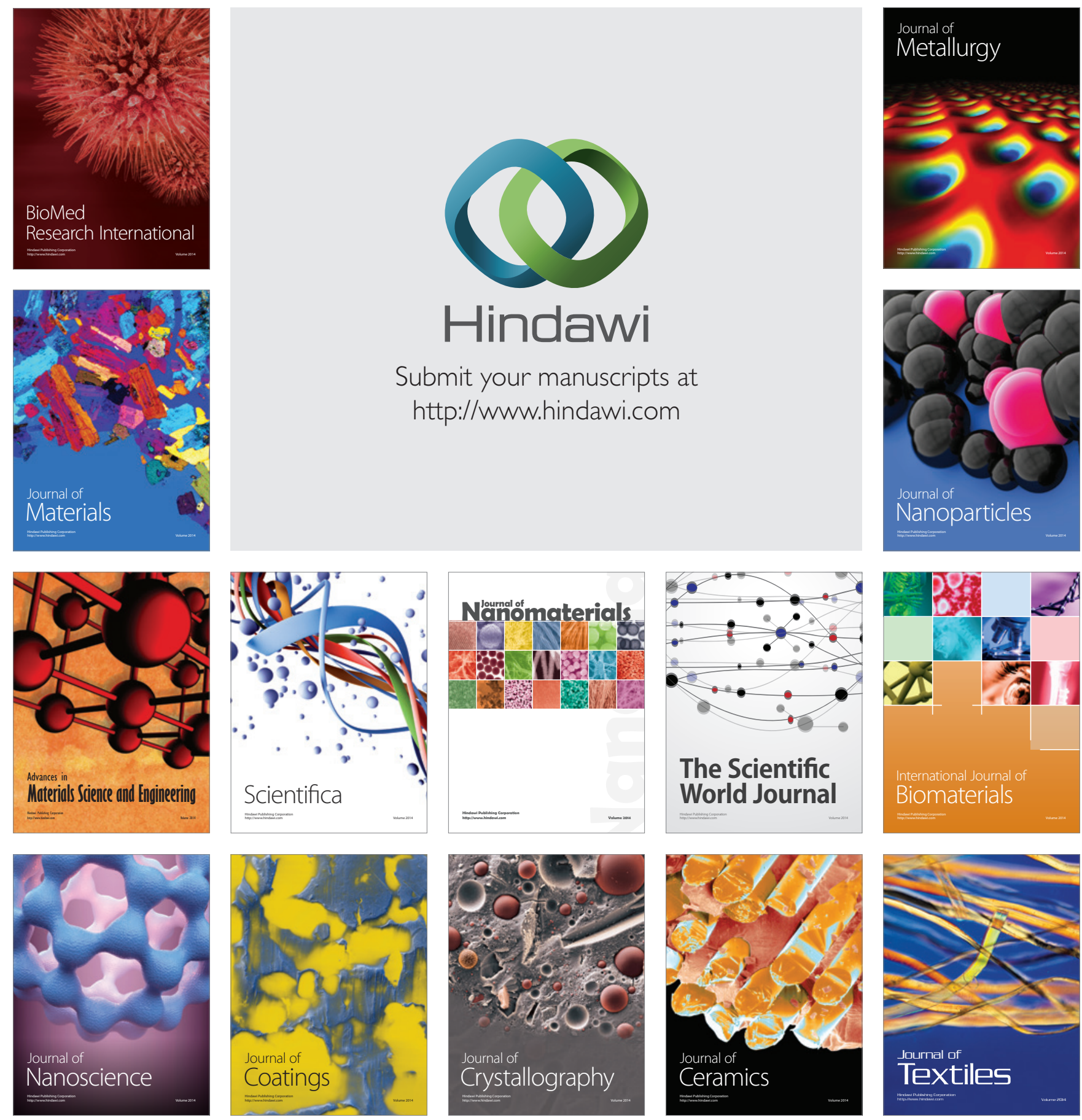